\title{
COHOMOLOGY OF KOSZUL-VINBERG ALGEBROIDS AND POISSON MANIFOLDS, I
}

\author{
MICHEL NGUIFFO BOYOM \\ UMR 5030 CNRS, Département de Mathématiques, Université Montpellier 2 \\ 34095 Montpellier Cedex 5, France \\ E-mail: boyom@math.univ-montp2.fr
}

\begin{abstract}
We introduce a cohomology theory of Koszul-Vinberg algebroids. The relationships between that cohomology and Poisson manifolds are investigated. We focus on the complex of chains of superorders [KJL1]. We prove that symbols of some sort of cycles give rise to so called bundlelike Poisson structures. In particular we show that if $E \rightarrow M$ is a transitive Koszul-Vinberg algebroid whose anchor is injective then a Koszul-Vinberg cocycle $\theta$ whose symbol has non-zero skew symmetric component defines a transversally Poissonian symplectic foliation in $M$.
\end{abstract}

1. Background material. Let $\mathcal{A}$ be a real algebra whose multiplication map is denoted by

$$
(a, b) \rightarrow a b .
$$

Given three elements $a, b, c$ of $\mathcal{A}$ their associator in $\mathcal{A}$ is the quantity

$$
(a, b, c)=a(b c)-(a b) c .
$$

Definition 1.1. A real algebra $\mathcal{A}$ is called a Koszul-Vinberg algebra if its associator map satisfies the identity

$$
(a, b, c)=(b, a, c) .
$$

N.B. Koszul-Vinberg algebras are also called left symmetric algebras [NB1], [PA].

Let $\mathcal{A}$ be a Koszul-Vinberg algebra and let $W$ be a real vector space with two bilinear maps

$$
\begin{aligned}
& \mathcal{A} \times W \rightarrow W: \quad(a, w) \rightarrow a w \\
& W \times \mathcal{A} \rightarrow W: \quad(w, a) \rightarrow w a .
\end{aligned}
$$

2000 Mathematics Subject Classification: Primary 22A22, 53B05, 53C12, 53D17; Secondary 17B55, $17 \mathrm{~B} 63$.

The paper is in final form and no version of it will be published elsewhere. 
We will set the following: given $a \in \mathcal{A}$ and $w \in W$

$$
\begin{aligned}
& (a, b, w)=a(b w)-(a b) w, \\
& (a, w, b)=a(w b)-(a w) b, \\
& (w, a, b)=w(a b)-(w a) b .
\end{aligned}
$$

Definition 1.2. A vector space equiped with two bilinear maps (2) is called a KoszulVinberg module of $\mathcal{A}$ if the following identities hold for any $a, b \in \mathcal{A}$ and $w \in W$ :

$$
\begin{aligned}
& (a, b, w)=(b, a, w), \\
& (a, w, b)=(w, a, b) .
\end{aligned}
$$

Given a Koszul-Vinberg algebra $\mathcal{A}$ and a Koszul-Vinberg module $W$ of $\mathcal{A}$, one of the following spaces:

$$
\begin{gathered}
J(\mathcal{A})=\{c \in \mathcal{A} /(a, b, c)=0, \forall a \in \mathcal{A}, \forall b \in \mathcal{A}\} \\
J(W)=\{w \in W /(a, b, w)=0, \forall a \in \mathcal{A}, \forall b \in \mathcal{A}\} .
\end{gathered}
$$

The subspace $J(\mathcal{A}) \subset \mathcal{A}$ is a subalgebra of $\mathcal{A}$ and the induced multiplication map is associative. In general the vector subspace $J(W)$ is not invariant under the actions (2).

Examples of Koszul-Vinberg algebras and their modules

$\left(e_{1}\right)$ Every associative algebra is a Koszul-Vinberg algebra.

$\left(e_{2}\right)$ Let $(M, D)$ be a locally flat manifold, [KJL3]; then the vector space $\Gamma(T M)$ of smooth vector fields on $M$ is a Koszul-Vinberg algebra; its multiplication map is defined by

$$
(X, Y) \rightarrow X Y=D_{X} Y .
$$

$\left(e_{3}\right)$ Given a locally flat manifold $(M, D)$ let $W$ be the vector space of real valued smooth functions on $M$. For any $f \in W$ and $X \in \Gamma(T M)$ we define $X f \in W$ and $f X \in W$ by putting

$$
(X f)(x)=<d f, X>(x), \quad(f X)(x)=0 \in \mathbb{R} .
$$

With the above operations $W$ becomes a Koszul-Vinberg module of $\mathcal{A}=\Gamma(T M)$.

Given a Koszul-Vinberg algebra $\mathcal{A}$ and two Koszul-Vinberg modules of $\mathcal{A}$, called $V$ and $W$, let $\operatorname{Hom}(W, V)$ be the vector space of linear maps from $W$ to $V$. We consider the following actions of $\mathcal{A}$ in $\operatorname{Hom}(W, V)$ : let $\theta \in \operatorname{Hom}(W, V), a \in \mathcal{A}, w \in W$ then we set

$$
(a \theta)(w)=a(\theta(w))-\theta(a w), \quad(\theta a)(w)=(\theta(w)) a .
$$

Under the actions defined in (4) the vector space $\operatorname{Hom}(W, V)$ becomes a KoszulVinberg module of $\mathcal{A}$. More generally the vector space $\operatorname{Hom}\left(\oplus^{q} W, V\right)$ of $q$-linear mappings from $W$ to $V$ is a Koszul-Vinberg module of $\mathcal{A}$ under the following actions: let $\theta \in$ $\operatorname{Hom}\left(\oplus^{q} W, V\right), a \in \mathcal{A}$ and $w_{1}, \ldots, w_{q} \in W$, we set

$$
\begin{gathered}
(a \theta)\left(w_{1}, \ldots, w_{q}\right)=a\left(\theta\left(w_{1}, \ldots, w_{q}\right)\right)-\sum_{1 \leq j \leq q} \theta\left(\ldots a w_{j}, \ldots, w_{q}\right), \\
(\theta a)\left(w_{1}, \ldots, w_{q}\right)=\left(\theta\left(w_{1}, \ldots, w_{q}\right)\right) a .
\end{gathered}
$$

Let $q$ be a positive integer every pair $\left(j, w_{0}\right)$ where $j$ is a non-negative integer with $j \leq q$ and $w_{0} \in W$ will define a linear map from $\operatorname{Hom}\left(\otimes^{q}, V\right)$ to $\operatorname{Hom}\left(\otimes^{q-1} W, V\right)$, called $e_{j}\left(w_{0}\right)$. Let $\theta \in \operatorname{Hom}\left(\otimes^{q} W, V\right)$ then $e_{j}\left(w_{0}\right) \theta \in H o m\left(\otimes^{q-1} W, V\right)$ is defined by

$$
\left(e_{j}\left(w_{0}\right) \theta\right)\left(w_{1}, \ldots, w_{q-1}\right)=\theta\left(w_{1}, \ldots, w_{j-1}, w_{0}, w_{j}, . . w_{q-1}\right) .
$$


The linear map $e_{j}\left(w_{0}\right)$ commutes with the right action of $\mathcal{A}$, viz

$$
\left(e_{j}\left(w_{0}\right) \theta\right) a=e_{j}\left(w_{0}\right)(\theta a) .
$$

Thus the notation $e_{j}\left(w_{0}\right) \theta a$ will be well defined.

We are now in a position to recall the definition of the complex

$$
\ldots \rightarrow C^{q}(\mathcal{A}, W) \stackrel{\delta_{q}}{\rightarrow} C^{q+1}(\mathcal{A}, W) \rightarrow \ldots
$$

Let $\mathcal{A}$ be a Koszul-Vinberg algebra and let $W$ be a Koszul-Vinberg module of $\mathcal{A}$. For each positive integer $q$ we set

$$
C^{q}(\mathcal{A}, W)=H o m\left(\otimes^{q} \mathcal{A}, W\right)
$$

and for $q=0$ we set

$$
C^{0}(\mathcal{A}, W)=J(W)
$$

Then the graded vector space

$$
C(\mathcal{A}, W)=\oplus_{q \geq 0} C^{q}(\mathcal{A}, W)
$$

is a cochain complex whose boundary operator is defined by

$$
\begin{gathered}
\delta_{0}: C^{0}(\mathcal{A}, W) \rightarrow C^{1}(\mathcal{A}, W), \quad\left(\delta_{0} w\right)(a)=-a w+w a, \\
\delta_{q}: C^{q}(\mathcal{A}, W) \rightarrow C^{q+1}(\mathcal{A}, W), \\
(\delta \theta)\left(a_{1}, \ldots, a_{q+1}\right)=\sum_{j \leq q}(-1)^{j}\left\{\left(a_{j} \theta\right)\left(. . \hat{a}_{j} . . a_{q+1}\right)+\left(e_{q}\left(a_{j}\right) \theta a_{q+1}\left(. . \hat{a}_{j} . ., \hat{a}_{q+1}\right)\right\}\right.
\end{gathered} .
$$

The family $\left(\delta_{q}\right)_{q}$ satisfies the following identity

$$
\delta_{q+1} \delta_{q}=0 .
$$

The $q^{\text {th }}$ cohomology space of the cochain complex $C(\mathcal{A}, W)$ is denoted by $H^{q}(\mathcal{A}, W)$. We have

$$
H^{q}(\mathcal{A}, W)=\operatorname{ker}\left(\delta_{q}\right) / \operatorname{im}\left(\delta_{q-1}\right)
$$

for $q>0$ and

$$
H^{0}(\mathcal{A}, W)=\operatorname{ker}\left(\delta_{0}\right) .
$$

ExAmple. Let $(M, D)$ be a locally flat manifold and let $\mathcal{A}=\Gamma(T M)$ be the corresponding Koszul-Vinberg algebra. Regarding $\mathcal{A}$ as a Koszul-Vinberg module of itself the subspace $J(\mathcal{A})$ consists of affine vector fields. Thus $\operatorname{ker}\left(\delta_{0}\right)$ is the subspace of locally linear vector fields. One sees that in general $H^{0}(\mathcal{A}, \mathcal{A})$ will be non-trivial; e.g. if $(M, D)$ is the real flat torus then $\operatorname{dim} H^{0}(\mathcal{A}, \mathcal{A})=\operatorname{dim} M$. On the other hand we have $H^{1}(\mathcal{A}, \mathcal{A})=0,\left[\mathrm{NB}_{3}\right]$.

2. Koszul-Vinberg algebroids and coalgebroids. Let $M$ be a smooth manifold and $E$ a vector bundle over $M$. The space of smooth sections of $E$ is denoted by $\Gamma(E)$.

Definition 2.1. A Koszul-Vinberg algebroid over $M$ is a vector bundle $E$ over $M$ with a bundle map $a: E \rightarrow T M$, called the anchor map, such that

$\left(P_{1}\right) \Gamma(E)$ is a Koszul-Vinberg algebra;

$\left(P_{2}\right)$ The anchor $a: \Gamma(E) \rightarrow \Gamma(T M)$ satisfies the following identities: $\forall f \in C^{\infty}(M, \mathbb{R})$, $\forall s \in \Gamma(E), \forall s^{\prime} \in \Gamma(E)$

$$
(f s) s^{\prime}=f\left(s s^{\prime}\right), \quad s\left(f s^{\prime}\right)=f\left(s s^{\prime}\right)+<d f, a(s)>s^{\prime} .
$$


REMARK. It follows from conditions (P1) and (P2) that the anchor map is a homomorphism of the associated Lie algebras.

Examples of Koszul-Vinberg algebroids

$\left(e_{1}\right)$ The tangent bundle of a locally flat manifold $(M, D)$ is a Koszul-Vinberg algebroid. Its anchor is Identity map; given two sections of $T M$, called $X, Y$ then

$$
X Y=D_{X} Y .
$$

$\left(e_{2}\right)$ Let $\mathcal{F}$ be an affine foliation in a smooth manifold $M$ and let $E_{\mathcal{F}}$ be the tangent bundle of $\mathcal{F}$ in $T M$. Since each leaf of $\mathcal{F}$ is a locally flat manifold $E_{\mathcal{F}}$ is a Koszul-Vinberg algebroid over $M$.

$\left(e_{3}\right)$ Each completely integrable system in an $m$-dimensional symplectic manifold $(M, \omega)$ gives rise to an action of $\mathbb{R}^{m}$ in $M$. The orbits of that action are locally flat manifolds; thus every completely integrable system will generate a Koszul-Vinberg algebroid.

$\left(e_{4}\right)$ Given a lagrangian foliation $\mathcal{F}$ in a symplectic manifold $(M, \omega)$ one defines a Koszul-Vinberg algebroid $E$ as in $\left(e_{2}\right)$. If $s, s^{\prime} \in \Gamma(E)$ then $s s^{\prime}$ is defined by the relation

$$
\iota\left(s s^{\prime}\right) \omega=L_{s} \iota\left(s^{\prime}\right) \omega
$$

where $\iota\left(s^{\prime}\right)$ is the inner product by $s^{\prime}$ and $L_{s}$ is the Lie derivation w.r.t. $s$. The multiplication in $\Gamma(E)$ given by $(6)$ induces a locally flat structure in each leaf of $\mathcal{F}$.

Now given a Koszul-Vingberg algebroid $E$ whose anchor map is injective, it is natural to ask whether the locally flat structure of leaves of $E$ extends to a locally flat structure in $M$. The notion of Koszul-Vinberg co-algebroid together with cochain complex (5) help to study the extension that we just raised, $[\mathrm{NBW}]$ (see also $[\mathrm{KI}]$ for the notion of partial connection).

Definition 2.2. Given a Koszul-Vinberg algebroid $E \rightarrow M$, a Koszul-Vinberg coalgebroid of $E$ is a vector bundle $N \rightarrow M$ together with a bundle map $\alpha: N \rightarrow T M$ satisfying the following conditions:

$\left(c_{1}\right) \Gamma(N)$ is a Koszul-Vinberg algebra.

$\left(c_{2}\right)$ There exists a linear map $j: \Gamma(T M) \rightarrow \Gamma(N)$ such that the sequence

$$
\Gamma(E) \stackrel{a}{\rightarrow} \Gamma(T M) \stackrel{j}{\rightarrow} \Gamma(N) \rightarrow 0
$$

is exact and $j \circ \alpha(s)=s, \forall s \in \Gamma(N)$.

$\left(c_{3}\right)$ Let $s, s^{\prime}$ be elements of $\Gamma(N)$ and $f \in C^{\infty}(M, \mathbb{R})$; then

$$
(f s) s^{\prime}=f\left(s s^{\prime}\right)
$$

and if $\langle d f, a(\sigma)\rangle=0$ for every $\sigma \in \Gamma(E)$ then

$$
s\left(f s^{\prime}\right)=f\left(s s^{\prime}\right)+<d f, \alpha(s)>s^{\prime} .
$$

EXAMPLE. Let $\mathcal{F}$ be a locally flat foliation which is a transversally affine foliation at the same time. Then the Koszul-Vinberg algebroid $E_{\mathcal{F}}$ corresponding to $\mathcal{F}$ admits a Koszul-Vinberg coalgebroid, [NBW].

Indeed let $\mathcal{L}$ be the sheaf of locally linear sections of $E_{\mathcal{F}}$, i.e. $s \in \mathcal{L}$ iff $s^{\prime} s=0$, $\forall s^{\prime} \in \Gamma\left(E_{\mathcal{F}}\right)$. We consider the quotient vector bundle $T M / E_{\mathcal{F}}$. Since $\mathcal{F}$ is transversally 
affine the space of smooth sections of $N=T M / E_{\mathcal{F}}$ admits a structure of Koszul-Vinberg algebra (every germ of submanifold which is transverse to $\mathcal{F}$ is a germ of affine manifold). Thus $\Gamma(N)$ admits a Koszul-Vinberg algebra structure. Let us write

$$
J(N)=J(\Gamma(N)) .
$$

Then $C^{\infty}(M, \mathbb{R}) J(N)=\Gamma(N)$. Using a riemannian metric on $M$ one constructs a section

$$
\alpha: N \rightarrow T M
$$

of the exact sequence

$$
0 \rightarrow E_{\mathcal{F}} \stackrel{a}{\rightarrow} T M \stackrel{j}{\rightarrow} N \rightarrow 0
$$

where $j$ is the canonical projection.

In $[\mathrm{NBW}]$ we have used the Lie algebra

$$
\mathcal{A}=\operatorname{norm}(\mathcal{L}) \cap j^{-1}(J(N))
$$

to study the extension problem of the locally flat structure of $\mathcal{F} ; \operatorname{norm}(\mathcal{L})$ is the normalizer of $\mathcal{L}$ in the Lie algebra $\Gamma(T M)$.

Remark that every Koszul-Vinberg algebroid $E$ gives rise to a Lie algebroid $E_{L}$; the total space of $E_{L}$ is $E$; for $s$ and $s^{\prime}$ in $\Gamma\left(E_{L}\right)$ the bracket is defined by

$$
\left[s, s^{\prime}\right]=s s^{\prime}-s^{\prime} s .
$$

The anchor map of $E$ satisfies the identity

$$
a\left(\left[s, s^{\prime}\right]\right)=\left[a(s), a\left(s^{\prime}\right)\right] .
$$

Indeed let $s, s^{\prime}, s^{\prime \prime}$ be elements of $\Gamma(E)$ and $f \in C^{\infty}(M, \mathbb{R})$, then

$$
\left[s, s^{\prime}\right]\left(f s^{\prime \prime}\right)=\left(s s^{\prime}\right)\left(f s^{\prime \prime}\right)-\left(s^{\prime} s\right)\left(f s^{\prime \prime}\right)=s\left(s^{\prime}\left(f s^{\prime \prime}\right)\right)-s^{\prime}\left(s\left(f s^{\prime \prime}\right)\right)
$$

and property $\left(P_{2}\right)$ of definition 2.1 implies that

$$
<d f, a\left(\left[s, s^{\prime}\right]\right)>=a(s)\left(a\left(s^{\prime}\right) f\right)-a\left(s^{\prime}\right)(a(s) f),
$$

where $a(s) f=<d f, a(s)>$.

3. Real cohomology of Koszul-Vinberg algebroids. Let $E \rightarrow M$ be a KoszulVinberg algebroid. The vector space $W=C^{\infty}(M, \mathbb{R})$ is a Koszul-Vinberg module of $\mathcal{A}=\Gamma(E)$. The left action and the right action are defined by

$$
(s f)(x)=<d f, a(s)>, \quad(f . s)(x)=0,
$$

where $a$ is the anchor map of $E$.

We will focus on the cochain complex

$$
\ldots \rightarrow C^{q}(\mathcal{A}, W) \stackrel{\delta_{q}}{\rightarrow} C^{q+1}(\mathcal{A}, W) \rightarrow \ldots
$$

The $q^{t h}$ cohomology space of $(8)$ is denoted by $H^{q}(E, \mathbb{R})$, i.e. $H^{q}(E, \mathbb{R})=H^{q}(\mathcal{A}, W)$.

Definition 2.3. The vector space $H^{q}(E, \mathbb{R})$ is called the $q^{t h}$ cohomology space of the Koszul-Vinberg algebroid $E \rightarrow M$.

ExAmPLE. Let $E$ be a regular Koszul-Vinberg algebroid whose anchor map is denoted by $a$. Then $a(E)$ defines a foliation on $M$. A function $f$ belongs to $J(W)$ iff $L_{a(s)} \circ$ 
$L_{a\left(s^{\prime}\right)}(f)=0$ for arbitrary sections $s, s^{\prime}$ of $E$. Thus if the anchor is injective then $J(W)$ consists of smooth functions which are affine along each leaf of $a(E)$. Since $H^{0}(E, \mathbb{R})=$ $\operatorname{ker}\left(\delta_{0}\right)$ we see that $H^{0}(E, \mathbb{R})$ is just the vector space of first integrals of $a(E)$.

THEOREM 3.1. If a regular Koszul-Vinberg algebroid $E \rightarrow M$ admits a dense leaf then $\operatorname{dim} H^{0}(E, \mathbb{R})=1$.

4. Koszul-Vinberg algebroids and Poisson manifolds. To every Koszul-Vinberg algebroid $E$ we attach the following new Koszul-Vinberg algebroid

$$
\mathcal{E}=E \times \mathcal{R}
$$

where $\mathcal{R}$ is the trivial vector bundle $\mathcal{R}=: M \times \mathbb{R}$. We identify $\Gamma(\mathcal{R})$ with the associative algebra $C^{\infty}(M, \mathbb{R})$ of smooth real valued functions on $M$. Thus we will identify $\Gamma(\mathcal{E})$ with $\Gamma(E) \times C^{\infty}(M, \mathbb{R})$ as well.

Henceforth $\Gamma(\mathcal{E})$ is an algebra whose multiplication is

$$
(s, f)\left(s^{\prime}, f^{\prime}\right)=\left(s s^{\prime}, f f^{\prime}+<d f^{\prime}, a(s)>\right) .
$$

It is easy to see that $(9)$ endows $\Gamma(\mathcal{E})$ with a structure of Koszul-Vinberg algebra. Morever if $g \in C^{\infty}(M, \mathbb{R})$ then we have

$$
(g(s, f))\left(s^{\prime}, f^{\prime}\right)=g\left((s, f)\left(s^{\prime}, f^{\prime}\right)\right)
$$

and

$$
(s, f)\left(g\left(s^{\prime}, f^{\prime}\right)\right)=g\left((s, f)\left(s^{\prime}, f^{\prime}\right)\right)+<d g, a(s)>\left(s^{\prime}, f^{\prime}\right) .
$$

Naturally the anchor map of $\mathcal{E}$ is defined by

$$
a_{\epsilon}(s, f)=a(s)
$$

where $a$ is the anchor of $E \rightarrow M$. The Koszul-Vinberg algebra $\Gamma(\mathcal{E})$ is the semi-product

$$
\Gamma(E) \times C^{\infty}(M, \mathbb{R}) .
$$

Now let $V$ be a vector space, let $r$ be a non-negative integer; we will put

$$
T^{r}(V)=\otimes^{r} V \text {. }
$$

Henceforth we are concerned with the cochain complex

$$
\ldots \rightarrow C^{q}(\mathcal{G}, W) \stackrel{\delta_{q}}{\rightarrow} C^{q}(\mathcal{G}, W) \rightarrow \ldots
$$

where $\mathcal{G}$ is the Koszul-Vinberg algebra $(9)$ and $W=C^{\infty}(M, \mathbb{R})$. For each non-negative integer $q$ the vector space $C^{q}(\mathcal{G}, W)$ is bigraded

$$
C^{q}(\mathcal{G}, W)=\oplus_{r+s=q} C^{r, s}(\mathcal{G}, W)
$$

with

$$
C^{r, s}(\mathcal{G}, W)=\operatorname{Hom}\left(T^{r} A \otimes T^{s} W, W\right),
$$

$r$ and $s$ being non-negative integers.

The boundary operator $\delta_{q}$ goes from $C^{r, s}(\mathcal{G}, W)$ to the $\operatorname{direct} \operatorname{sum} C^{r+1, s}(\mathcal{G}, W) \oplus$ $C^{r, s+1}(\mathcal{G}, W)$. Thus we will equip the cohomology space $H^{q}(\mathcal{G}, W)$ with the bigradation

$$
H^{q}(\mathcal{G}, W)=\oplus_{r+s=q} H^{r, s}(\mathcal{G}, W)
$$


with

$$
H^{r, s}(\mathcal{G}, W)=\frac{\operatorname{ker}\left(\delta_{q}: C^{r, s}(\mathcal{G}, W) \rightarrow C^{r+1, s}(\mathcal{G}, W) \oplus C^{r, s+1}(\mathcal{G}, W)\right)}{\delta_{q-1}\left(C^{q-1}(\mathcal{G}, W)\right) \cap C^{r, s}(\mathcal{G}, W)} .
$$

Naturally one sees that

$$
\delta_{q-1}\left(C^{q-1}(\mathcal{G}, W)\right) \cap C^{r, s}(\mathcal{G}, W)=\delta_{q-1}\left(C^{r-1, s}(\mathcal{G}, W)+C^{r, s-1}(\mathcal{G}, W)\right) \cap C^{r, s}(\mathcal{G}, W) .
$$

We will develop the analogue of the complex of differential forms of superorder introduced by Jean-Louis Koszul, [KJL2].

To begin with, let $\xi \in \mathcal{G}$, for a non-negative integer $k$ and $x \in M j_{x}^{k} \xi$ is the $k^{t h}$ jet at $x$ of $\xi \in \mathcal{G}$. We will present $j_{x}^{k} \xi$ by

$$
j_{x}^{k} \xi=\left(d_{x}^{1} \xi, . ., d_{x}^{l} \xi, . ., d_{x}^{k} \xi\right)
$$

where $d_{x}^{l} \xi$ is the $l^{\text {th }}$ differential at $x$ of the section $\xi \in \Gamma(\mathcal{E})$.

Definition [KJL2]. A cochain $\theta \in C^{q}(\mathcal{G}, W)$ is of order $\leq k$ if at every $x \in M$ and for $\xi_{1}, . ., \xi_{q} \in \mathcal{G}$ the value at $x$ of $\theta\left(\xi_{1}, . . \xi_{q}\right)$ depends on, $j_{x}^{k} \xi_{1}, . . j_{x}^{k} \xi_{q}$.

Let $I=\left(i_{1}, . ., i_{q}\right)$ be a $q$-tuple of non-negative integers such that $i_{l} \leq k$. Given a $q$-cochain $\theta \in C^{q}(\mathcal{G}, W)$ of order $\leq k$, we set

$$
\theta^{I}\left(\xi_{1}, . ., \xi_{q}\right)(x)=\theta\left(d_{x}^{i_{1}} \xi_{1}, . ., d_{x}^{i_{q}} \xi_{q}\right) .
$$

Since $\theta$ is $q$-multilinear (11) makes sense.

Thus every $\theta \in C^{q}(\mathcal{G}, W)$ which is of order $\leq k$ will be decomposed as follows

$$
\theta\left(\xi_{1}, . ., \xi_{q}\right)=\sum_{I} \theta^{I}\left(\xi_{1}, . ., \xi_{q}\right)
$$

where $I=\left(i_{1}, . ., i_{q}\right)$ with $0 \leq i_{1}, . ., i_{q} \leq k$.

We call $\theta^{I}$ the component of type $I$ of $\theta$.

The following definition is crucial for the forthcoming applications.

Definition 4.1 Given a cochain of order $\leq k$, say $\theta \in C^{q}(\mathcal{G}, W)$, then its component of type $(k, . ., k)$ is called the symbol of $\theta$.

Notice that the symbol of $\theta$ may be zero.

Proposition [NB4]. The symbol $\sigma(\theta)$ of every $q$-cocycle $\theta \in C^{0, q}(\mathcal{G}, W)$ is $\delta_{q}$ closed and satisfies the identity

$$
s \sigma(\theta)=0
$$

for any arbitrary element $s \in \Gamma(E)$.

We recall that

$$
\left(s(\sigma(\theta))\left(\xi_{1}, . ., \xi_{q}\right)=a(s)\left(\sigma(\theta)\left(\xi_{1}, . ., \xi_{q}\right)\right)-\sum_{j \leq q} \sigma(\theta)\left(\ldots s \xi_{j}, . . \xi_{q}\right) .\right.
$$

For every non-negative interger $r, H^{r, 0}(\mathcal{G}, W)=0$. (That phenomenon may be explained by using an appropriate spectral sequence.)

We are going now to relate symbols of so called Koszul-Vinberg cocycle to Poisson manifolds structures.

We will deal with the vector spaces $C^{r, s}(\mathcal{G}, W)$ such that $r s=0$. For instance $C^{0,2}(\mathcal{G}, W)$ may contain Poisson tensors as well as Jacobi tensors. 
On the other hand let us suppose that the Koszul-Vinberg algebroid $E \rightarrow M$ has an injective anchor map. Then Riemannian metrics or symplectic structures on the vector bundle $E \rightarrow M$ give rise to elements of $C^{2,0}(\mathcal{G}, W)$.

Definition 4.2. (i) A cochain $\theta \in C^{2}(\mathcal{G}, W)$ is called a Koszul-Vinberg cochain if for arbitrary elements $\xi_{1}, \xi_{2}, \xi_{3}$ of $\mathcal{G}$ one has

$$
\left(\xi_{1}, \xi_{2}, \xi_{3}\right)_{\theta}=\left(\xi_{2}, \xi_{1}, \xi_{3}\right)_{\theta}
$$

where

$$
\left(\xi_{1}, \xi_{2}, \xi_{3}\right)_{\theta}=\theta\left(\xi_{1}, \theta\left(\xi_{2}, \xi_{3}\right)\right)-\theta\left(\theta\left(\xi_{1}, \xi_{2}\right), \xi_{3}\right)
$$

(ii) $\theta \in C^{2}(\mathcal{G}, W)$ is a Koszul-Vinberg cocycle if $\delta \Pi_{\theta}=\delta . \theta=0$ and $\left(\xi_{1}, \xi_{2}, \xi_{3}\right)_{\theta}=$ $\left(\xi_{2}, \xi_{1}, \xi_{3}\right)_{\theta}$.

Definition 4.2 makes sense because $W$ may be regarded as a subspace of $\mathcal{G}$.

Every Koszul-Vinberg cochain $\theta \in C^{2}(\mathcal{G}, W)$ defines a Koszul-Vinberg algebra structure whose multiplication is given by

$$
\xi_{1} \xi_{2}=\theta\left(\xi_{1}, \xi_{2}\right)
$$

Therefore we define in $\mathcal{G}$ a new Lie algebra structure called $\mathcal{G}_{\theta}$, whose bracket is given by

$$
\left[\xi_{1}, \xi_{2}\right]_{\theta}=\theta\left(\xi_{1}, \xi_{2}\right)-\theta\left(\xi_{2}, \xi_{1}\right) .
$$

Before continuing we will recall some differential geometry structures related to the cohomology of Koszul-Vinberg algebroids.

Definition 4.3. (i) A Poisson foliation in a manifold $M$ is a foliation $\mathcal{F}$ whose leaves are Poisson manifolds.

(ii) A transversally Poisson foliation in $M$ is a foliation whose sheaf of basic functions is a sheaf of Poisson algebra.

Part (ii) in definition 4.3 has the following meaning: the sheaf of local first integrals of $\mathcal{F}$ admits a Lie algebra bracket

$$
(f, g) \rightarrow\{f, g\}
$$

such that

$$
\{f, g h\}=g\{f, h\}+\{f, g\} h .
$$

Let us go back to considerations regarding the complex

$$
. . \rightarrow C^{q}(\mathcal{G}, W) \stackrel{\delta_{q}}{\rightarrow} C^{q+1}(\mathcal{G}, W) \rightarrow . .
$$

which is defined by a Koszul-Vinberg algebroid $E \rightarrow M$. The following claim is easily verified $[\mathrm{KM}]$. Let $A$ be an associative and commutative algebra and let $C(A, A)$ be its Hochschild complex. Given any 2-cocycle $\theta \in C^{2}(A, A)$ and any $\xi \in A$, then the linear map say $\theta_{\xi}$

$$
\zeta \rightarrow \theta(\xi, \zeta)-\theta(\zeta, \xi)
$$


is a derivation of the algebra $A$. This elementary result has deep consequences; for example given a smooth manifold $M$ with a start product in $C^{\infty}(M, \mathbb{R})$, say

$$
f * g=f g+\sum_{k>0} h^{k} B_{k}(f, g)
$$

the bilinear map $B_{1}: C^{\infty}(M, \mathbb{R})^{2} \rightarrow C^{\infty}(M, \mathbb{R})$ is a cocycle of the Hochschild complex of $C^{\infty}(M, \mathbb{R})$. One deduces that $B_{1}$ is a bidifferential operator of order 1 whose skew symmetric component defines a Poisson manifold structure on $M,[\mathrm{KM}]$. The same claim doesn't hold in the cohomology theory of Koszul-Vinberg algebras. For instance in a Koszul-Vinberg algebra $\mathcal{A}$ the multiplication map

$$
(a, b) \rightarrow a b
$$

is an exact cocycle of $C(A, A)$, but the linear map

$$
b \rightarrow a b-b a
$$

for a fixed $a$ is a derivation of $\mathcal{A}$ iff $a \in J(\mathcal{A})$. That makes relevant the theorem which is stated below.

Let $E \rightarrow M$ be a Koszul-Vinberg algebroid and let $C(\mathcal{G}, W)$ be the complex associated to $\mathcal{G}=\Gamma(\mathcal{E})$.

TheOREM I $\left[\mathrm{NB}_{4}\right]$. Let $\theta \in C^{0,2}(\mathcal{G}, W)$ be a cocycle of order $\leq k$. If the skew symmetric component of the symbol $\sigma(\theta)$ is non-zero, then $k=1$.

An important consequence of theorem is the following statement:

THEOREM II [NB4]. The skew symmetric component of the symbol $\sigma(\theta)$ of every Koszul-Vinberg cocycle $\theta \in C^{0,2}(\mathcal{G}, W)$ is a Poisson tensor.

Now let us assume the Koszul-Vinberg algebroid $E \rightarrow M$ to be regular. Then $E$ defines a foliation $E_{\mathcal{F}}$ in $M$. Given any Koszul-Vinberg cocycle $\theta \in C^{0,2}(\mathcal{G}, W)$ of order $\leq k$ we denote by $\Pi_{\theta}$ the skew symmetric component of $\sigma(\theta)$. The following corollary follows directly from theorem II.

Corollary 4.4. Every germ of submanifold in $M$ which is normal to $\mathcal{F}_{E}$ is a germ of Poisson submanifold of $\left(M, \Pi_{\theta}\right)$. In particular if $\mathcal{F}_{E}$ is simple then the quotient manifold $M / \mathcal{F}_{E}$ admits a Poisson manifold structure $\left(M / \mathcal{F}_{E}, \tilde{\Pi}_{\theta}\right)$ such that the canonical projection from $M$ to $M / \mathcal{F}_{E}$ is a Poisson morphism from $\left(M, \Pi_{\theta}\right)$ to $\left(M / \mathcal{F}_{E}, \tilde{\Pi}_{\theta}\right)$.

Considering the case of Koszul-Vinberg algebroids with injective anchor maps, we see that such algebroids define locally flat foliations in their base manifolds. Thus we can state the following

THEOREM III [NB4]. Let $E \rightarrow M$ be a Koszul-Vinberg algebroid whose anchor map is injective. If $E$ is transitive, then every Koszul-Vinberg cocycle $\theta \in C^{0,2}(\mathcal{G}, W)$ defines a regular Poisson structure on $M$.

Remark that $W$ being a Koszul-Vinberg submodule of $\mathcal{G}$ every Koszul-Vinberg cochain $\tilde{\theta} \in C^{2}(\mathcal{G}, \mathcal{G})$ induces a Koszul-Vinberg cochain $\theta \in C^{0,2}(\mathcal{G}, W)$. 
5. The Koszul-Vinberg analogues of star product. Let $M$ be a smooth manifold and let $W$ be the vector space $C^{\infty}(M, \mathbb{R})$ endowed with its natural structure of associative and commutative algebra.

Given a start product in $W$, say

$$
f * f^{\prime}=f f^{\prime}+\sum_{k>0} h^{k} B_{k}\left(f, f^{\prime}\right)
$$

it is well known that the skew symmetric component of $B_{1}$ is a Poisson tensor on $M$, $[\mathrm{KM}]$. Regarding theorem II a natural question arises: does the same phenomenon persist in Koszul-Vinberg algebra structures.

Henceforth we will consider a Koszul-Vinberg algebroid $E \rightarrow M$. As before we denote by $\mathcal{G}$ the vector space of smooth sections of the Wihtney sum $E \oplus \mathcal{R}$. We consider the multiplication already defined by $(9)$, i.e. for $\xi=(s, f), \xi^{\prime}=\left(s^{\prime}, f^{\prime}\right)$

$$
\xi \xi^{\prime}=\left(s s^{\prime}, f f^{\prime}+<d f^{\prime}, a(s)>\right)
$$

where $a$ is the anchor map of $E$. Let $h$ be some parameter; we will focus on the familly of multiplication in $\mathcal{G}$

$$
\xi *_{h} \xi^{\prime}=\xi \xi^{\prime}+\sum_{k>0} h^{k} \theta_{k}\left(f, f^{\prime}\right)
$$

with $\theta_{k} \in C^{2}(\mathcal{G}, \mathcal{G})$. We suppose the multiplication $(12)$ to satisfy Definition 1.1 , viz

$$
\left(\xi_{1}, \xi_{2}, \xi_{3}\right) *_{h}=\left(\xi_{2}, \xi_{1}, \xi_{3}\right) *_{h}
$$

for elements $\xi_{1}, \xi_{2}, \xi_{3}$ of $\mathcal{G}$. Thus we obtain a family $\mathcal{G}_{h}$ of Koszul-Vinberg algebras. The coefficient $\theta_{1}$ is a cocycle of the complex $C(\mathcal{G}, \mathcal{G})$.

Each Koszul-Vinberg algebra $\mathcal{G}_{h}$ give rise to a Lie algebra whose bracket is given by

$$
\left[\xi, \xi^{\prime}\right]_{h}=\xi *_{h} \xi^{\prime}-\xi^{\prime} *_{h} \xi=\left[\xi, \xi^{\prime}\right]+\sum_{k>0} h^{k} \Lambda_{k}\left(\xi, \xi^{\prime}\right)
$$

with $\Lambda_{k}\left(\xi, \xi^{\prime}\right)=\theta_{k}\left(\xi, \xi^{\prime}\right)-\theta_{k}\left(\xi^{\prime}, \xi\right)$.

In order that the pair $\left(E \oplus \mathcal{R}, \mathcal{G}_{h}\right)$ define a Koszul-Vinberg algebroid with the same anchor map $a$ as the pair $(E \oplus \mathcal{R}, \mathcal{G})$ it is necessary that

$$
a\left(\left[\xi, \xi^{\prime}\right]_{h}\right)=a([\xi, \xi]) .
$$

Thus we must have

$$
a\left(\sum_{k>0} h^{k} \Lambda_{k}\left(\xi, \xi^{\prime}\right)\right)=0 .
$$

Therefore we see that for every positive integer $k$ one has $a\left(\Lambda_{k}\left(\xi, \xi^{\prime}\right)\right)=0$. On the other hand recall that $W$ is a two-sided ideal of the Koszul-Vinberg algebra $\mathcal{G}$ whose multiplication is (9). Then the $W$-component of the cocycle $\theta_{1}$ is a $W$-valued 2-cocycle of the cochain complex $C(\mathcal{G}, W)$. By assuming that the map $a$ is also the anchor map of the pair

$$
\left(E \oplus \mathcal{R}, \mathcal{G}_{h}\right)
$$

we deduce from the condition

$$
\xi\left(f *_{h} \xi^{\prime}\right)=f\left(\xi * \xi^{\prime}\right)+<d f, a(\xi)>\xi^{\prime}
$$


that the chains $\theta_{k}$ are of order zero, that is to say that each $\theta_{k}$ is tensorial. This phenomenon is in contrast to the case of star products in the associative and commutative algebra $C^{\infty}(M, \mathbb{R})$.

To end the present paper we deduce from (13) the following statement.

Proposition 5.1. Let $E \rightarrow M$ be a Koszul-Vinberg algebroid whose anchor map is injective. Suppose that the associated algebroid $E \oplus \mathcal{R}$ admits a one parameter family of deformations $\left(E \oplus \mathcal{R}, \mathcal{G}_{h}\right)$ whose multiplication is

$$
\xi *_{h} \xi^{\prime}=\xi \xi^{\prime}+\sum_{k>0} h^{k} \theta_{k}\left(\xi, \xi^{\prime}\right) .
$$

Then the coefficients $\theta_{k}$ are symmetric chains of the cochain complex $C(\mathcal{G}, \mathcal{G})$.

\section{References}

[GM] M. Gerstenhaber, Deformation of rings and algebras, Ann. of Math. 79 (1964), 59-103.

[KM] M. Kontsevich, Deformation quantization of Poisson manifolds, Preprint Alg./ 9709040.

[KJL1] J-L. Koszul, Homologie des complexes des formes différentielles d'ordre supérieur, Ann. Scient. Ec. Norm. Sup 7 (1964), 139-159.

[KJL2] J-L. Koszul, Déformations des connections localement plates, Ann. Inst. Fourier 18 (1968), 103-114.

[KJL3] J-L. Koszul, Crochet de Schouten-Nijenhuis et cohomologie, in: Elie Cartan et les mathématiques d'aujourd'hui, Soc. Math. de France, Astérisque, 1985, 257-271.

[KI] J. Kubarski, Bott's vanishing theorem for regular Lie algebroids, Trans. Amer. Math. Soc. 348 (1996), 2151-2167.

[LP] P. Libermann, Sur les plongements des fibrés principaux et groupoïdes différentiables, Séminaire An. globale, Montreal, 1969, 7-108.

[L-M] P. Libermann and C. M. Marle, Symplectic Geometry and Analytical Mechanics, Reidel, Dordrecht, 1987.

[MCM] C. M. Marle, Symplectic manifolds, dynamical systems and hamiltonian mechanics, in: Differential Geometry and Relativity in Honour to A. Lichnerowicz, Reidel, Dordrecht, 1976.

[NA] A. Nijenhuis, Sur une classe de propriétés communes à quelques types différents d'algèbres, Enseign. Math. 14 (1949), 225-277.

[NB1] M. Nguiffo Boyom, Algèbres à associateur symétrique et algèbres de Lie réductives, Thèse Doctorat TC-Fac. Scienc. Grenoble, 1968.

[NB2] M. Nguiffo Boyom, Structures localement plates isotropes des groupes de Lie, Ann. Sc. Norm. Sup. Pisa 20 (1993), 91-131.

[NB3] M. Nguiffo Boyom, The homology theory of Koszul-Vinberg algebra, submitted.

[NB4] M. Nguiffo Boyom, Cotangent Koszul-Vinberg algebroids and Poisson manifolds, in preparation.

[NB-W] M. Nguiffo Boyom and R. Wolak, Affine structure and KV homology, in preparation.

[PA] A. M. Perea, Flat left symmetric connections adapted to the automorphism structure of Lie groups, J. Diff. Geom. 16 (1981), 445-474.

[PW] W. S. Piper, Algebraic deformation theory, J. Diff. Geom. 1 (1967), 133-168. 
[RM] M. S. Raghunathan, Deformations of linear connections and Riemannian manifolds, J. Math. Mech. 13 (1964), 97-123.

[VI1] I. Vaisman, Lectures on the Geometry of Poisson Manifolds, Progr. Math. 118, Birkhäuser, Berlin, 1994.

[VI2] I. Vaisman, On the geometry quantization of Poisson manifolds, J. Math. Physics 32 (1991), 3339-3345.

[VJ] J. Vey, Déformations du crochet de Poisson sur une variété symplectique, Comm. Math. Helv. 50 (1975), 421-454.

[WA] A. Weinstein, The local structure of Poisson manifolds, J. Diff. Geom. 18 (1983), 523-557. 